\title{
Specific Features of Primary Central Nervous System Lymphoma in Comparison with Glioblastoma on Conventional MRI
}

\author{
Chao Xiang ${ }^{1}$, Qianxue Chen ${ }^{1, *}$ and Yunfei Zha ${ }^{2}$ \\ ${ }^{1}$ Department of Neurosurgery, Renmin Hospital of Wuhan University, Wuhan, Hubei, China \\ ${ }^{2}$ Department of Radiology, Renmin Hospital of Wuhan University, Wuhan, Hubei, China \\ "Corresponding author: Department of Neurosurgery, Renmin Hospital of Wuhan University, Minkang Building, Zhangzhidong Rd., Wuchang District, Wuhan, Hubei, China. \\ Tel: +86-13607141618, Email: xiangchao1981@sina.com
}

Received 2018 May 15; Revised 2018 October 01; Accepted 2018 November 12.

\begin{abstract}
Background: The treatments of primary central nervous system lymphoma (PCNSL) and glioblastoma (GBM) are different; therefore, accurate diagnosis is crucial for the therapeutic planning. Although there is overlap of features on conventional magnetic resonance (MR) images between PCNSL and GBM, the PCNSL has specific features, which are different from those of GBM on conventional MR images.

Objectives: The current study aimed at characterizing specific features of PCNSL by comparing them with conventional MR images of GBM, and improving diagnostic accuracy at the time of initial presentation.

Patients and Methods: The current study retrospectively evaluated the conventional MR images of 21 immunocompetent patients with PCNSL and 13 patients with GBM pathologically proven in Renmin Hospital of Wuhan University, Wuhan, China from January 2014 to March 2017. All patients were subjected to non-contrast and contrast enhanced MRI. Tumor location, quantity, and morphology, as well as common and specific features and patterns, peritumoral edema, and hemorrhage were recorded and compared.

Results: Twenty-one patients with PCNSL exhibited 44 lesions, and 13 patients with GBM exhibited 16 lesions. PCNSL affected both infra- and supratentorial regions, while GBM only affected supratentorial regions. Deep white matter, basal ganglia, periventricular areas, and the corpus callosum were the most common sites in PCNSL. Homogeneous enhancement was presented in 95.2\% of PCNSL, and all GBM lesions showed heterogeneous enhancement. PCNSLs had various appearances, whereas most of the GBMs (81.3\%) had a mass-like appearance. Differences in intratumoral hemorrhage $(\mathrm{P}<0.001)$, necrotic or cystic components $(\mathrm{P}<0.001)$, and intratumoral vascular enhancement $(\mathrm{P}<0.001)$ between PCNSL and GBM were statistically significant. "Notch sign" and open-ring enhancement were specific features for PCNSL.

Conclusion: PCNSL has specific features, patterns, and locations. These features and patterns can be helpful to differentiate PCNSL from GBM at the time of initial presentation.
\end{abstract}

Keywords: Extraventricular, Glioblastoma, Lymphoma, MRI

\section{Background}

Primary central nervous system lymphoma (PCNSL) is a rare malignant tumor that accounts for $2 \%-6 \%$ of all primary brain tumors and $1 \%-2 \%$ of all non-Hodgkin lymphomas (1). The incidence of PCNSL increased over recent years in immunocompetent patients (2-4). Glioblastoma (GBM) is the most frequent and severe glioma subtype that accounts for about $50 \%$ of diffuse gliomas (5). PCNSL is very chemo- and radiosensitive, and surgical resection does not improve the outcome (6). In contrast, the primary treatment for GBM is surgical resection followed by radio- and chemotherapy $(5,7)$. Since the treatments for PCNSL and GBM differ, accurate diagnosis is very important at the time of initial presentation. Since conventional MR features of
PCNSL and GBM overlap, misdiagnosis can be made at the time of initial presentation.

\section{Objectives}

Since conventional MR features of PCNSL and GBM overlap, the current study aimed at characterizing specific features and patterns that differentiate PCNSL from GBM on conventional MRI.

\section{Patients and Methods}

Conventional MR images of 21 patients with PCNSL (14 males,seven females, age range of 24 - 67 years, mean age 
of $51 \pm 15$ years) and 13 patients with GBM (seven males, six females, age range of 30 - 68 years, mean age of 50 \pm 12 years) examined in Renmin Hospital of Wuhan University, Wuhan from January 2014 to March 2017 were reviewed retrospectively. All the patients with PCNSL were pathologically proven (14 patients by stereotactic biopsy and seven patients by surgical excision) diffuse large B-cell lymphoma (DLBCL) and all patients with GBM were pathologically proven by surgical excision (World Health Organization [WHO] grade IV). All the patients with PCNSL had no evidence of systemic lymphoma (no abnormalities were observed in bow marrow aspiration and blood test, and no lymphadenopathy was observed in body CT scan), immunocompromised disease, or use of an immunosuppressant. The current retrospective study obtained verbal consent from the review board and ethics committee of our institution.

All patients were subjected to non-contrast and contrast enhancement that included axial T1-weighted imaging (T1WI) and T2-weighted imaging (T2WI), sagittal fluidattenuated inversion recovery (FLAIR), and intravenous gadolinium contrast enhancement (axial, coronal, and sagittal) with a 1.5-T MRI scanner (GE Signa HDi, USA) within two weeks before surgical excision or biopsy. MRI parameters were as follows: T1WI (repetition time [TR] $2000 \mathrm{~ms}$, echo time [TE] $10 \mathrm{~ms}$, inversion time [TI] $800 \mathrm{~ms}$ ), T2WI (TR 3000 ms, TE 80 ms), FLAIR (TR 11000 ms, TE 120 ms, TI $2800 \mathrm{~ms}$ ), thickness $6 \mathrm{~mm}$, interval $10 \mathrm{~mm}$, field-of- view (FOV) $24 \times 24 \mathrm{~cm}$; contrast agent gadolinium diethylenetriaminepentaacetic acid (Gd-DTPA), dosage $0.2 \mathrm{mM} / \mathrm{kg}$. All image data were transferred to a picture archiving and communication system (PACS) system and analyzed by two experienced radiologists blinded to the pathology results. Lesion location, number, morphology, specific features, enhanced patterns, peritumoral edema, and hemorrhage were recorded. Features, patterns, and degree of edema are presented in Table 1. Signal intensity on T1WI and T2WI was stratified as hyper-, iso-, and hypointense compared with gray matter. Categorical values were analyzed using the chi-squared test. A P value $<0.05$ was considered statistically significant. Analyses were performed with SPSS version 13.0 .

\section{Results}

Patient data and MR findings of PCNSL and GBM at the initial presentation are summarized in Table 2. Differences in gender predominance $(\mathrm{P}=0.491)$ and mean age \pm standard deviation (SD) (PCNSL $51 \pm 15$ years, GBM $50 \pm 12$ years, $\mathrm{P}=0.866$ ) between PCNSL and GBM groups were not statistically significant. A total of 44 lesions were PCNSL. Of these, $52.4 \%$ of cases were single lesions, and only $9.5 \%$

\begin{tabular}{ll}
\hline Table 1. Definitions of Features and Patterns \\
\hline Features and patterns & Definition \\
\hline Notch sign & Focal depressed margin of lesion \\
\hline Butterfly sign or mirror image & $\begin{array}{l}\text { Lesion simultaneously involves } \\
\text { bilateral white matter and the corpus } \\
\text { callosum showing a symmetric pattern }\end{array}$ \\
\hline Intratumoral vascular flow void & $\begin{array}{l}\text { Intratumoral linear hypointensity on } \\
\text { T2WI and/or CE caused by flow void } \\
\text { effect }\end{array}$ \\
\hline Necrotic or cystic formation & $\begin{array}{l}\text { Hypointense on T1WI, hyperintense on } \\
\text { T2WI, and no enhancement on CE }\end{array}$ \\
\hline Hemorrhage & Hyperintense on T1WI and T2WI \\
\hline Degree of perilesional edema & \\
\hline Mild & Emax $\leq 1 / 2$ Dmax \\
\hline Moderate & $1 / 2$ Dmax $<$ Emax $\leq$ Dmax \\
\hline Marked & Emax $>$ Dmax \\
\hline
\end{tabular}

Abbreviations: CE, contrast enhancement; Dmax, maximum diameter of tumor; Emax, maximum diameter of perilesional edema; T2WI, T2 weighted image; T1WI, T1 weighted image

were in the infratentorial region. A total of 16 lesions were GBM. Of these, $84.6 \%$ of cases were single lesions, and all 16 lesions were in the supratentorial region. Differences in type and location of lesions were not statistically significant between the PCNSL and GBM groups ( $\mathrm{P}=0.075$ and $\mathrm{P}=0.513$, respectively). PCNSL lesions exhibited worm-like (50\%), nodular (29.5\%), mass-like (9.1\%), and patchy infiltrative (11.4\%) appearances (Table 2 ). PCNSL preferentially occurred in deep white matter, basal ganglia, and periventricular areas (36.4\% in the frontal lobe, $18.2 \%$ in basal ganglia, $11.4 \%$ in the periventricular area, $11.4 \%$ in the corpus callosum, 9.1\% in the parietal lobe, $4.5 \%$ in the cerebellum, $2.3 \%$ in the occipital lobe; the frontal lobe and corpus callosum were affected concomitantly in $6.8 \%$ of cases). The frontal lobe was the most common site, and three cases showed concomitant involvement of the frontal lobe and corpus callosum with a "butterfly sign" or "mirror image" (Figure 1A - C). One PCNSL case (4.8\%) was heterogeneously enhanced due to central necrosis (Figure 1D - F), while the other lesions were homogeneously enhanced. Part of one subcortical lesion was wrapped around the cortex giving the appearance of a crescent (Figure 1G). A "notch" sign (Figure $1 \mathrm{H}$ ) was observed in $25 \%$ of cases, and open-ring enhancement(Figure 1J and Figure 2A-B) was observed in $4.5 \%$ of cases. No intratumoral vascular flow void was observed on T2WI and/or contrast enhancement (CE). Intratumoral vascular enhancement (IVE) (Figure 2C - F) was observed in $28.6 \%$ of cases, and all had a worm-like appearance. Perilesional edema presented in all cases (mild: $14.3 \%$, moderate: $23.8 \%$, marked: $61.9 \%$ ). No signs of hemorrhage were detected in PCNSL. 

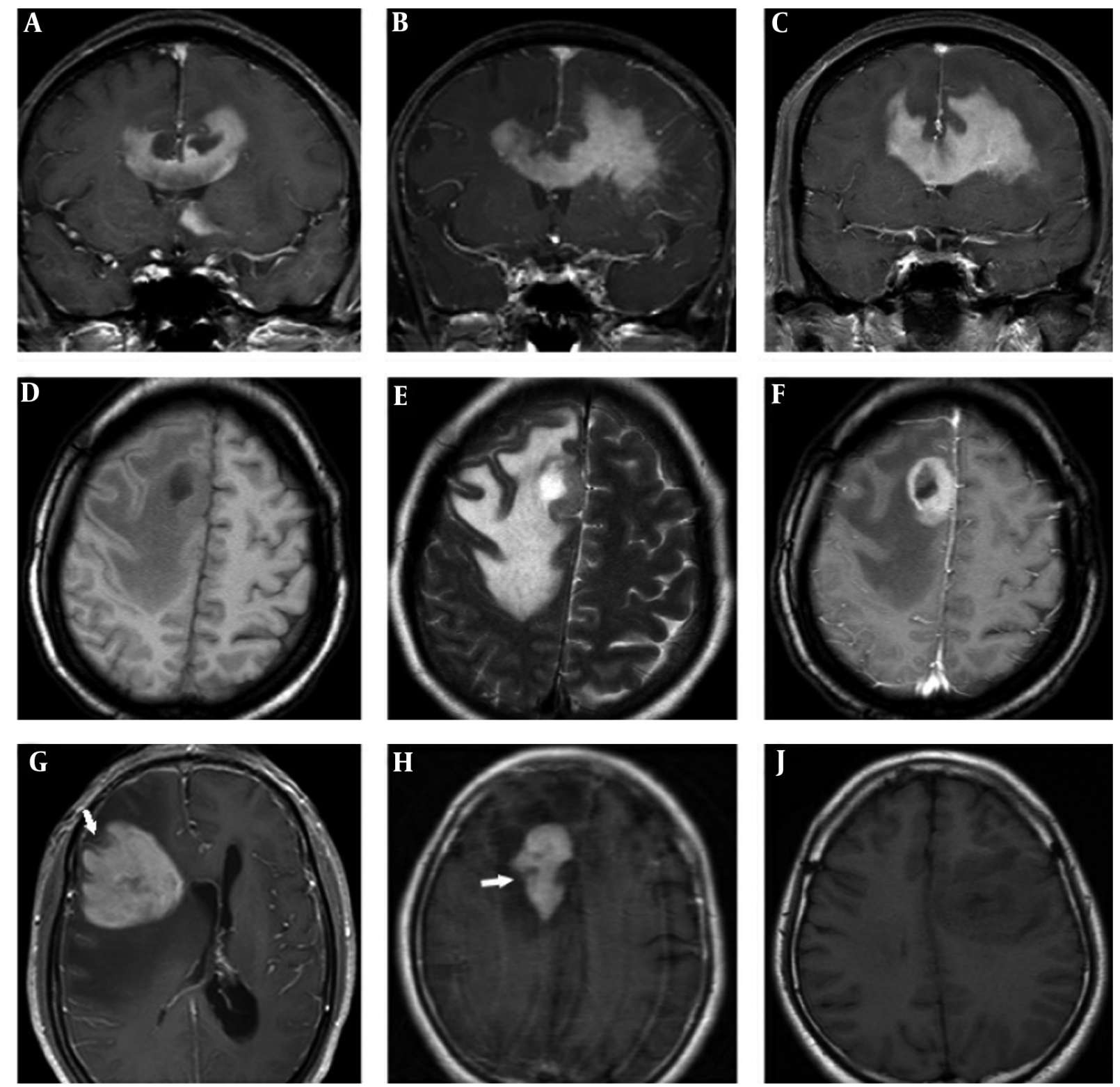

Figure 1. Bilateral frontal lobe and corpus callosum involvement showing the "butterfly sign" or "mirror image" (A-C). Right frontal lobe primary central nervous system lymphoma (PCNSL) lesions with intratumoral necrosis (D-F). Axial T1-weighted image (D), T2-weighted image (E) and contrast enhanced image (F). Subcortical PCNSL lesion wrapped around the cortex, giving the appearance of a crescent (G). A PCNSL lesion with a "notch sign" (white arrow) in the right frontal lobe (H). A PCNSL lesion on axial T1-weighted image (J).

GBM lesions exhibited nodular, mass-like, and patchy infiltrative morphology and none appeared worm-like. GBM lesions were observed in all supratentorial lobes (56.3\% in the frontal lobe, $25 \%$ in the occipital lobe, $12.5 \%$ in the parietal lobe, and $6.3 \%$ in the temporal lobe), and the frontal lobe was the most common site. Overall, $81.3 \%$ of lesions had a mass-like appearance, $12.5 \%$ had a patchy infiltrative appearance, and $6.2 \%$ had a nodular appearance.
Only one case (7.7\%) exhibited intratumoral hemorrhage (Table 2). A necrotic or cystic component was detected in $84.6 \%$ of cases and IVE (Figure $2 \mathrm{G}-\mathrm{J}$ ) was observed in $92.3 \%$ of cases. Perilesional edema presented in all lesions (mild: 30.8\%, moderate: $38.4 \%$, marked: $30.8 \%$ ) and no "notch sign" was observed in GBM. Ring enhancement presented in 75\% of GBM cases. An intratumoral vascular flow void (Figure $3 \mathrm{~A}-\mathrm{E}$ ) on T2WI and/or CE presented in all GBM cases. 

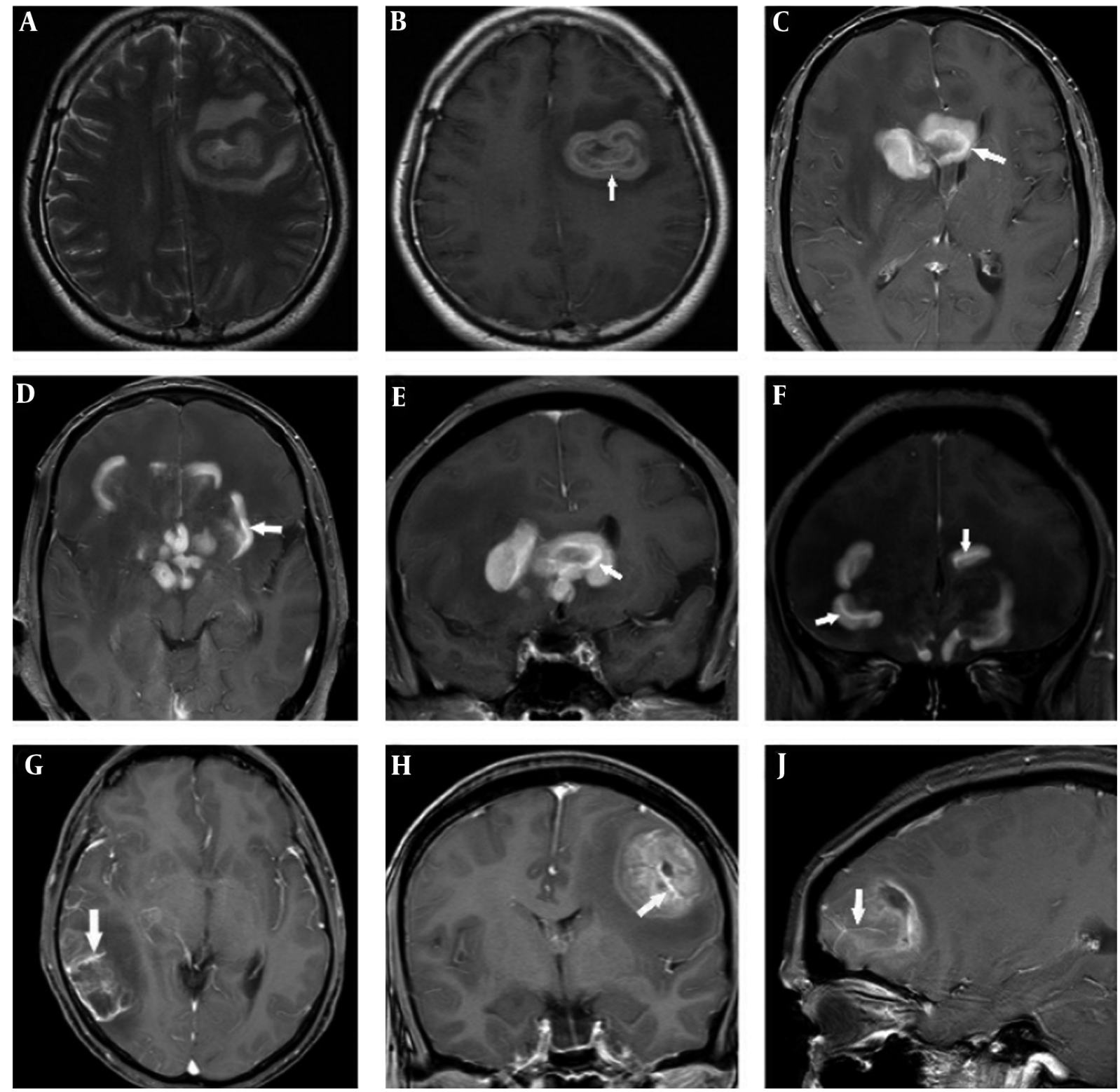

Figure 2. A primary central nervous system lymphoma (PCNSL) lesion on axial T2-weighted image (A). A PCNSL lesion with open-ring enhancement and intratumoral vascular enhancement(white arrow) in the left frontal lobe (B). Worm-like PCNSL lesions with single and regular intratumoralvascular enhancement (white arrows)(C-F). Glioblastoma (GBM) lesions with multiple irregular intratumoral vascular enhancement (white arrows) (G-J).

Leptomeningeal involvement (Figure 3F and 3G) presented both in GBM and PCNSL subcortical lesions (five cases in GBM, one case in PCNSL). There were no signs of cranial nerve involvement in GBM and PCNSL.

\section{Discussion}

PCNSL and GBM are malignant brain tumors that can lead to a poor outcome and prognosis if correct treatments are not achieved at the time of initial presentation (4). PCNSL is very chemo- and radiosensitive, and surgical resection does not improve the outcome (6). In contrast, the primary treatment for GBM is surgical resection followed by radio- and chemotherapy $(5,7)$. Thus, correct diagnosis at initial presentation is crucial to choose the treatment (8). No specific MRI features are reported to diagnose PCNSL (9) and it is difficult to morphologically differentiate between PCNSL and GBM by MRI (5). However, the current study 

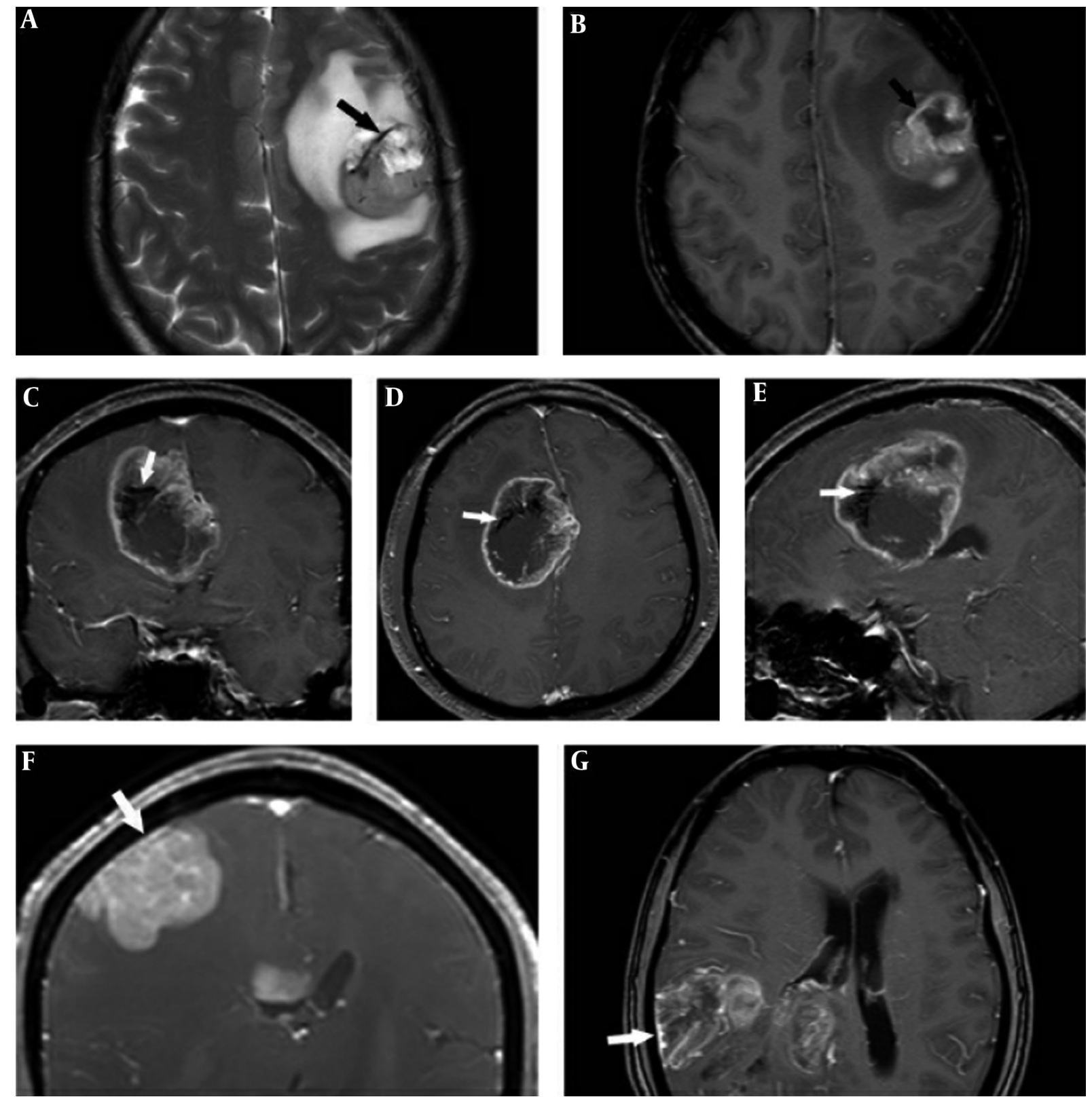

Figure 3. Glioblastoma (GBM) intratumoral vascular flow void (A-E), in a T2-weighted image (A). The same vessel showed contrast enhancement on contrast enhancement (CE) (B). Intratumoral vascular flow void on CE in a coronal image (C), axial image (D), and sagittal image (E). Subcortical primary central nervous system lymphoma (PCNSL) (F) and GBM (G) lesions with adjacent leptomeningeal involvement.

showed that PCNSL had specific features and patterns on conventional MR images that differed from those of GBM and may be helpful to differentiate the two types of tumor.

The mean age of patients with PCNSL at initial diagnosis was $51 \pm 15$ years, which was similar to those of previous reports (10). There was no significant differences in gender ratio $(\mathrm{P}=0.491)$ or mean age $(\mathrm{P}=0.866)$ between the $\mathrm{PCNSL}$ and GBM groups. PCNSL lesions presented both in infraand supratentorial regions, while GBM lesions were more likely to occur in supratentorial regions. However, this localization difference was not statistically significant $(\mathrm{P}=$ $0.513)$. The frontal lobe was the most common site affected by PCNSL and GBM. It is reported that $27 \%-49 \%$ of PCNSLs are in the frontal lobe $(1,10)$, and PCNSLs are more likely to 
Table 2. Patient Inclusion Data and MR Findings in PCNSL and GBM at the Time of Initial Presentation ${ }^{\mathrm{a}, \mathrm{b}}$

\begin{tabular}{|c|c|c|c|}
\hline & PCNSL & GBM & Pvalue \\
\hline Gender & & & 0.491 \\
\hline Male & 14 & 7 & \\
\hline Female & 7 & 6 & \\
\hline Mean age $(y)$ & $51 \pm 15$ & $50 \pm 12$ & 0.866 \\
\hline Total lesions & 44 & 16 & \\
\hline Type of lesions & & & 0.075 \\
\hline Single lesion & $11(52.40)$ & $11(84.60)$ & \\
\hline Multiple lesions & $10(47.60)$ & $2(15.40)$ & \\
\hline Location of lesions (per person) & & & 0.513 \\
\hline Infratentorial & $2(9.50)$ & $0(0)$ & \\
\hline Supratentorial & $19(90.50)$ & $13(100)$ & \\
\hline Type of enhancement (per person) & & & $<0.001^{\mathrm{C}}$ \\
\hline Heterogeneous & $1(4.80)$ & $13(100)$ & \\
\hline Homogeneous & $20(95.20)$ & $0(0)$ & \\
\hline \multicolumn{4}{|l|}{ Appearance (per lesion) } \\
\hline Worm-like & $22(50)$ & $0(0)$ & $<0.001^{\mathrm{C}}$ \\
\hline Nodular & $13(29.50)$ & $1(6.20)$ & 0.123 \\
\hline Mass-like & $4(9.10)$ & $13(81.30)$ & $<0.001^{\mathrm{c}}$ \\
\hline Patchy infiltrative & $5(11.40)$ & $2(12.50)$ & 1.000 \\
\hline \multicolumn{4}{|l|}{ Common features (per person) } \\
\hline Hemorrhage & $0(0)$ & $1(7.70)$ & $<0.001^{\mathrm{c}}$ \\
\hline Necrotic or cystic component & $1(4.80)$ & $11(84.60)$ & $<0.001^{\mathrm{c}}$ \\
\hline $\begin{array}{l}\text { Intratumoral vascular } \\
\text { enhancement }\end{array}$ & $6(28.60)$ & $12(92.30)$ & $<0.001^{\mathrm{c}}$ \\
\hline \multicolumn{4}{|l|}{ Perilesional edema (per person) } \\
\hline Mild & $3(14.30)$ & $4(30.80)$ & 0.387 \\
\hline Moderate & $5(23.80)$ & $5(38.40)$ & 0.451 \\
\hline Marked & $13(61.90)$ & $4(30.80)$ & 0.158 \\
\hline \multicolumn{4}{|l|}{ Specific features (per lesion) } \\
\hline Notch & $11(25)$ & $0(0)$ & $<0.001^{\mathrm{c}}$ \\
\hline $\begin{array}{l}\text { Ring or open-ring } \\
\text { enhancement }\end{array}$ & $2(4.50)$ & $12(75)$ & $<0.001^{\mathrm{C}}$ \\
\hline $\begin{array}{l}\text { Intratumoral vascular flow } \\
\text { void on } \mathrm{T} 2 \mathrm{WI} \text { and/or CE }\end{array}$ & $0(0)$ & $16(100)$ & $<0.001^{\mathrm{C}}$ \\
\hline
\end{tabular}

Abbreviations: $\mathrm{CE}$, contrast enhancement; GBM, glioblastoma; MR, magnetic resonance; PCNSL, primary central nervous system lymphoma, T2WI, T2 weighted image.

${ }^{a}$ Values are expressed as No. (\%) or mean \pm SD (standard deviation).

${ }^{\mathrm{b}}$ Nodular: maximum diameter of lesion $<3 \mathrm{~cm}$, mass-like: maximum diame-

ter of lesion $\geq 3 \mathrm{~cm}$

${ }^{\mathrm{c}}$ Level of significance at $\mathrm{P}<0.05$.

occur in deep white matter, basal ganglia, and periventricular areas $(1,10)$, consistent with the results of the current study.
Although superficial lesions of PCNSL are common (2), subcortical lesions are more frequent in GBM than PCNSL. Both PCNSL and GBM subcortical lesions can reach the surface of the brain, causing adjacent leptomeninges linear enhancement (Figure 3F - G). In the current study, leptomeningeal involvement abutting subcortical lesions was observed in five GBM cases and one PCNSL case. In addition, it was observed that some PCNSL subcortical lesions conformed to cortical morphology. The PCNSL subcortical lesions wrapped around the cortex, forming a crescent shape (Figure 1G). This feature suggested pliable and infiltrative properties. None of the GBM lesions showed this feature.

The parenchymal signal intensities of PCNSL and GBM on non-contrast enhanced images were non-specific due to similar organizational structures such as hypercellularity and a high nucleus/cytoplasm ratio. On CE imaging, all PCNSL lesions, except one lesion with central necrosis, were homogeneously enhanced. In contrast, all GBM lesions were heterogeneously enhanced. This difference was statistically significant (Table 2 ) and consistent with a previous study (5).

The different appearances and patterns on contrast enhanced images were analyzed (Table 2). Most PCNSL lesions had worm-like (50\%) and nodular (29.5\%) appearances, and almost all worm-like lesions were in periventricular or deep white matter areas. In contrast, most GBM lesions had a mass-like (81.3\%) appearance, and all lesions were in subcortical or white matter areas. No worm-like appearance was observed in GBM lesions. Only five and two patchy infiltrative lesions were observed in PCNSL and GBM cases, respectively, with no statistically significant difference. Two ring enhancement lesions were observed in PCNSL. One was caused by central necrosis, and the other was caused by a worm-like growth pattern that mimicked the openring enhancement pattern.

Hemorrhage and internal calcification are rarely observed in PCNSL lesions (6), but they are common in GBM (5). This contributes to the homogeneous signal intensity on non-contrast and contrast enhanced MR images of PCNSL. In the current study, none of the PCNSL lesions exhibited hemorrhage or calcification. In addition, hemorrhage was rarely observed in GBM lesions. Only one GBM lesion exhibited hemorrhage, which was inconsistent with the results of previous studies $(2,5)$. However, this result may be due to the small GBM cohort in the current study.

Perilesional edema implies disruption of the bloodbrain barrier and diffuse infiltration of tumor cells. A previous study reported that the degree of perilesional edema in PCNSL was less significant than those of high grade glioma and metastases (2). However, the current study results were inconsistent with this finding. In the current 
study patient cohort, perilesional edema in PCNSLs was moderate to marked, and there was no significant difference between PCNSL and GBM (Table 2). This discordance may be due to different definitions of degree of perilesional edema.

The current study identified the following specific features and patterns that demonstrate significant differences between PCNSL and GBM: (1) Notch sign. This sign was defined as a focal depressed margin of the lesion, and it was first reported by Zhang et al. (10). In the current cohort study, 25\% of PCNSL lesions manifested a "notch sign", which suggested an irregular growth pattern as well as pliable and infiltrative properties. None of the GBM lesions showed this pattern $(\mathrm{P}<0.001)$; (2) Necrotic or cystic component. A necrotic or cystic component is rare in PCNSL (11), but common in GBM (5), and the current study was consistent with this finding. Only one PCNSL case (4.7\%) exhibited intratumoral necrosis, while 11 GBM cases (84.6\%) exhibited a necrotic or cystic component. This difference was significant $(\mathrm{P}<0.001)$. However, intratumoral necrosis in PCNSL is considered common in T-cell lymphoma and immunodeficient patients (1); (3) Open-ring enhancement. This feature is considered a specific sign to differenciate large, contrast-enhancing, demyelinating lesions from neoplasms and infection (12). The ring of enhancement is thick and uniform in PCNSL rather than thin and non-uniform in atypical brain demyelination. This sign suggests pliable and infiltrative properties of PCNSL. Ring enhancement in PCNSL was reported in the literature (10), but it was more frequent in AIDS-related PCNSL than in non-AIDS PCNSL (1). Although one lesion exhibited openring enhancement and one case exhibited ring enhancement in PCNSL, none of the GBM lesions had this pattern; (4) Intratumoral flow void vessel. GBM and PCNSL display different vascular features. Functional MRI studies demonstrated that PCNSL had lower relative cerebral blood volume (rCBV) than GBM $(7,13,14)$. This difference can be explained by active angiogenesis of GBM that leads to more extensive vasculature in GBM than in PCNSL. In the current study, all GBM lesions manifested intratumoral flow void vessels on T2WI and/or CE images (Figure 3A - E), but none of PCNSL lesions presented this feature. IVE could be observed both in PCNSL and GBM lesions (Figure 2C - J). IVE was detected in $28.6 \%$ of PCNSL cases and $92.3 \%$ of GBM cases, and the difference was statistically significant $(\mathrm{P}<$ 0.001). IVE appeared differently in MRimages of PCNSL and GBM. IVE in PCNSL manifested as a single, uniform linear enhancement within the lesion and along the shape of the lesion. This feature was observed in all worm-like PCNSL lesions. In contrast, IVE in GBM manifested as multiple, nonuniform, irregular linear enhancements (Figure 2G - J).

The "mirror image" or "butterfly pattern" was defined as symmetrical lesions that involve the frontal lobe and genu of the corpus callosum. This pattern was observed in three patients with PCNSL but none of the patients with GBM. Since GBM is an infiltrative malignant tumor, the "butterfly pattern" was reported in GBM cases in the literature (15). Thus, the "mirror image" or "butterfly pattern" is a non-specific feature that cannot be used to differentiate PCNSL and GBM.

Functional MRI and histopathological studies demonstrated that PCNSL had higher vascular permeability and blood flow compared with GBM $(13,16)$. GBM shows vascular integrity despite endothelial proliferation, whereas PCNSL shows loss of vessel architecture, which promotes vascular disintegrity (13). This loss of vessel integrity leads to contrast agent leakage and retention in the interstitial space of PCNSL lesions, which exhibit moderate to marked enhancement.

PCNSL can involve cranial nerves, and cranial nerves can be the only involved site. According to published data, 15\% - 25\% of patients with PCNSL had ocular involvement (17). Malikova et al. (5), reported that $42.6 \%$ of PCNSL cases involved optic pathways, $5.6 \%$ of cases involved other cranial nerves, and one case exhibited solitary auditory nerve involvement without other lesions. Ohta et al. (17), reported a patient with PCNSL with ocular involvement. In the current study, no cranial nerves showed any signs of involvement. However, cranial nerve and leptomeningeal involvement are very common in secondary central nervous system (CNS) lymphoma (2). The current study had several limitations. First, the number of patients in the current cohort study was small. Only 21 PCNSL and 13 GBM cases were included. All the cases with PCNSL were of the diffuse large B-cell lymphoma (DLBCL) subtype. Therefore, selective bias could not be avoided. Second, although functional MRI (such as diffusion-weighted imaging, magnetic resonance spectroscopy, perfusion-weighted imaging) was useful to differentiate PCNSL and GBM when conventional MRI findings overlap (7), only some of the current study cases were examined by functional MRI techniques and a statistical comparison was not possible. A larger number of patients and wider application of advanced MRI techniques are needed for further studies.

In summary, PCNSLs can involve both infra- and supratentorial regions, and typical locations include the frontal lobe, basal ganglia, and periventricular white matter. The lesions can be worm-like, nodular, mass-like, or patchy in appearance with homogeneous enhancement. Due to pliability of PCNSLs, subcortical lesions can wrap around the cortex, giving rise to a crescent appearance. Open-ring enhancement, "notch sign", and homogeneous enhancement with regular IVE can be specific features to differentiate PCNSL from GBM. Although IVE is observed both 
in PCNSL and GBM, and there is no significant difference between them, IVE patterns differ in PCNSL and GBM images, which should be considered. Intratumoral vascular flow voids only manifest in GBM lesions. Necrotic or cystic components within the lesion cannot exclude PCNSL, but they are rare. Finally, the current study observed that PCNSL showed specific features and patterns on conventional MRI. Thus, conventional MRI, especially contrast enhanced MRI, is a reliable technique to diagnose PCNSL and GBM at the time of initial presentation.

\section{Footnotes}

Authors' Contributions: The authors contribute equally to this work.

Conflict of Interests: There are no conflict of interests among the authors or with other people or ognizations.

Ethical Considerations: This retrospective study obtained verbal consent from the review board and Ethics Committee of Renmin Hospital of Wuhan University.

Financial Disclosure: None declared.

Funding/Support: None declared.

\section{References}

1. Haldorsen IS, Kråkenes J, Krossnes BK, Mella O, Espeland A. CT and MR imaging features of primary central nervous system lymphoma in Norway, 1989-2003. AJNR Am J Neuroradiol. 2009;30(4):744-51. doi: 10.3174/ajnr.A1447. [PubMed: 19164442].

2. Haldorsen IS, Espeland A, Larsson EM. Central nervous system lymphoma: Characteristic findings on traditional and advanced imaging. AJNR Am J Neuroradiol. 2011;32(6):984-92. doi: 10.3174/ajnr.A2171. [PubMed: 20616176].

3. Ponzoni M, Kwee I, Mazzucchelli L, Ferreri AJ, Zucca E, Doglioni C, et al. A virtual tissue bank for primary central nervous system lymphomas in immunocompetent individuals. Pathobiology. 2007;74(4):264-9. doi: 10.1159/000104455. [PubMed: 17709970].

4. Takeuchi H, Matsuda K, Kitai R, Sato K, Kubota T. Angiogenesis in primary central nervous system lymphoma (PCNSL). J Neurooncol. 2007;84(2):141-5. doi: 10.1007/s11060-007-9363-x. [PubMed: 17406788].

5. Malikova H, Koubska E, Weichet J, Klener J, Rulseh A, Liscak R, et al. Can morphological MRI differentiate between primary central nervous system lymphoma and glioblastoma? Cancer Imaging. 2016;16(1):40. doi: 10.1186/s40644-016-0098-9. [PubMed: 27894359]. [PubMed Central: PMC5126849].

6. Mansour A, Qandeel M, Abdel-Razeq H, Abu Ali HA. MR imaging features of intracranial primary CNS lymphoma in immune competent patients. Cancer Imaging. 2014;14:22. doi: 10.1186/1470-7330-14-22. [PubMed: 25608570]. [PubMed Central: PMC4331827].
7. Choi YS, Lee HJ, Ahn SS, Chang JH, Kang SG, Kim EH, et al. Primary central nervous system lymphoma and atypical glioblastoma: Differentiation using the initial area under the curve derived from dynamic contrast-enhanced MR and the apparent diffusion coefficient. Eur Radiol. 2017;27(4):1344-51. doi: 10.1007/s00330-016-4484-2. [PubMed: 27436023].

8. Xu W, Wang Q, Shao A, Xu B, Zhang J. The performance of MR perfusion-weighted imaging for the differentiation of high-grade glioma from primary central nervous system lymphoma: A systematic review and meta-analysis. PLoS One. 2017;12(3). e0173430. doi: 10.1371/journal.pone.0173430. [PubMed: 28301491]. [PubMed Central: PMC5354292].

9. da Rocha AJ, Sobreira Guedes BV, da Silveira da Rocha TM, Maia Junior AC, Chiattone CS. Modern techniques of magnetic resonance in the evaluation of primary central nervous system lymphoma: Contributions to the diagnosis and differential diagnosis. Rev Bras Hematol Hemoter. 2016;38(1):44-54. doi: 10.1016/j.bjhh.2015.12.001. [PubMed: 26969774]. [PubMed Central: PMC4786762].

10. Zhang D, Hu LB, Henning TD, Ravarani EM, Zou LG, Feng XY, et al MRI findings of primary CNS lymphoma in 26 immunocompetent patients. Korean J Radiol. 2010;11(3):269-77. doi: 10.3348/kjr.2010.11.3.269. [PubMed: 20461180]. [PubMed Central: PMC2864853].

11. Coulon A, Lafitte F, Hoang-Xuan K, Martin-Duverneuil N, Mokhtari K, Blustajn J, et al. Radiographic findings in 37 cases of primary CNS lymphoma in immunocompetent patients. Eur Radiol.2002;12(2):329-40. doi: 10.1007/s003300101037. [PubMed: 11870430].

12. Masdeu JC, Quinto C, Olivera C, Tenner M, Leslie D, Visintainer P. Openring imaging sign: Highly specific for atypical brain demyelination. Neurology. 2000;54(7):1427-33. [PubMed: 10751251].

13. Kickingereder P, Sahm F, Wiestler B, Roethke M, Heiland S, Schlemmer HP, et al. Evaluation of microvascular permeability with dynamic contrast-enhanced MRI for the differentiation of primary CNS lymphoma and glioblastoma: Radiologic-pathologic correlation. AJNR Am J Neuroradiol. 2014;35(8):1503-8. doi: 10.3174/ajnr.A3915. [PubMed: 24722313].

14. Toh $\mathrm{CH}$, Wei $\mathrm{KC}$, Chang $\mathrm{CN}$, Ng SH, Wong HF. Differentiation of primary central nervous system lymphomas and glioblastomas: Comparisons of diagnostic performance of dynamic susceptibility contrast-enhanced perfusion MR imaging without and with contrastleakage correction. AJNR Am J Neuroradiol. 2013;34(6):1145-9. doi: 10.3174/ajnr.A3383. [PubMed: 23348763].

15. Bourekas EC, Varakis K, Bruns D, Christoforidis GA, Baujan M, Slone HW, et al. Lesions of the corpus callosum: MR imaging and differential considerations in adults and children. AJR Am J Roentgenol. 2002;179(1):251-7. doi: 10.2214/ajr.179.1.1790251. [PubMed: 12076946].

16. Yamashita K, Yoshiura T, Hiwatashi A, Togao O, Yoshimoto K, Suzuki SO, et al. Differentiating primary CNS lymphoma from glioblastoma multiforme: Assessment using arterial spin labeling, diffusionweighted imaging, and (1)(8)F-fluorodeoxyglucose positron emission tomography. Neuroradiology. 2013;55(2):135-43. doi: 10.1007/s00234012-1089-6. [PubMed: 22961074].

17. Ohta K, Sano K, Suzuki T, Hidaka E, Yoshida A, Kikuchi T. B cell clonality of primary central nervous system and primary intraocular lymphomas. Jpn J Ophthalmol. 2007;51(2):147-9. doi: 10.1007/s10384-0060406-1. [PubMed: 17401628]. 